\title{
New features of flat (4+1)-dimensional cosmological model with a perfect fluid in Gauss-Bonnet gravity
}

\author{
I.V. Kirnos \\ Tomsk State University of Control Systems and Radioelectronics, Tomsk, 634050 Russia \\ S.A. Pavluchenko \\ Special Astrophysical Observatory, Russian Academy of Sciences, Nizhnij Arkhyz, 369167 Russia \\ A.V. Toporensky \\ Sternberg Astronomical Institute, Moscow State University, Moscow, 119992 Russia \\ We investigated a flat multidimensional cosmological model in Gauss-Bonnet gravity in \\ presence of a matter in form of perfect fluid. We found analytically new stationary regimes \\ (these results are valid for arbitrary number of spatial dimensions) and studied their stability \\ by means of numerical recipes in $4+1$-dimensional case. In the vicinity of the stationary \\ regime we discovered numerically another non-singular regime which appears to be periodi- \\ cal. Finally, we demonstrated that the presence of matter in form of a perfect fluid lifts some \\ constraints on the dynamics of the $4+1$-dimensional model which have been found earlier. \\ PACS numbers: 04.20.Jb, 04.50.-h, 04.50.Kd, 98.80.-k
}

\section{INTRODUCTION}

Theories of gravity with the Gauss-Bonnet (GB) term as a correction to the Einstein term in the action have been actively investigated for more than 30 years [1 5$]$. This term appears as the first non-Einstein contribution in the extension of the General Relativity (GR) known as the Lovelock gravity [6] (the only theory that keeps the equations of motion to be the 2nd order differential equations), and string gravity [7]. In $3+1$ dimensions this term does not contribute to the equations of motion, and it can be important either in a combination with another fields (as it does in string theory) or in theories containing scalar function of the Gauss-Bonnet invariant (see, for example [8]; in this case we, however, have higher-order equations of motion).

For higher number of spatial dimensions the Gauss-Bonnet term is dynamically important. Moreover, in Lovelock gravity (in contrast with string gravity) this term is the only allowed nonEinstein contribution in $(4+1)$ and $(5+1)$ dimensions. This means that it is possible to study regimes where this term is not a small corrections to Einstein gravity, but equally important or 
even dominant.

In the end of 80th some important results in multidimensional cosmology with the Gauss-Bonnet term have been obtained including the analog of Kasner solution in a pure Gauss-Bonnet gravity 3]. Further studies reveal interesting differences between $(4+1)$-dimensional case (this is the lowest number of dimensions for GB term to contribute) and higher-dimensional cosmology. This includes differences in the form of power-law solutions [3, 10], noncontinuity between vacuum solutions and solutions with matter (in $(4+1)$ flat case with non-zero matter density $\rho$ the limit $\rho \rightarrow 0$ may not coincide with vacuum solutions [12]). Some of the differences can be extended to higher-order Lovelock corrections (at least in a flat case [13]). Other example of particularity of $(4+1)$ case is severe fine-tuning needed for smooth evolution from high density (where GB term dominates) to low density (when Einstein gravity is restored) - such evolution requires three of four Hubble parameters to be equal with high precision [14]. This result have been obtained numerically in the theory which includes both GB and Einstein terms, so abovementioned results on dynamics with matter [12] (founded for a pure GB gravity) does not directly applicable to this case. Nevertheless, as we shell see, nonzero matter density can alter corresponding dynamics significantly.

The structure of the manuscript is as follows: first, we write down equations of motion for the model considered. Then, we analytically investigate stationary case and demonstrate exact solutions found. Then we switch to numerical studies and confirm by means of numerical recipes the reality of the previously found solutions as well as describe new oscillationary regime. Also we demonstrate (also via numerical methods) that the presence of matter in form of a prefect fluid lifts some constraints on the dynamics of the model considered. Finally, in the Conclusions we summarize the results found.

\section{EQUATIONS OF MOTION}

We consider a flat anisotropic metric in (4+1)-dimensional space-time. We are dealing with Einstein-Gauss-Bonnet gravity, and non-vacuum space-time. Lagrangian of this theory have a form

$$
\mathcal{L}=R+\alpha \mathcal{L}_{2}+\mathcal{L}_{M}
$$

where $R$ is Ricci scalar, $\mathcal{L}_{M}$ is the Lagrangian of matter fields and $\mathcal{L}_{2}$ 


$$
\mathcal{L}_{2}=R_{\mu \nu \alpha \beta} R^{\nu \mu \alpha \beta}-4 R_{\mu \nu} R^{\mu \nu}+R^{2}
$$

is the Gauss-Bonnet Lagrangian.

We are working in the flat background, so the metric we considering has a form

$$
g_{\mu \nu}=\operatorname{diag}\left\{-1, a^{2}(t), b^{2}(t), c^{2}(t), d^{2}(t)\right\}
$$

We use perfect fluid with the equation of state $p=w \rho$ as a matter source; after varying action obtained from (11) using the metric above and perfect fluid as a matter field, one can obtain the following equations of motion - there are $n$ dynamical equations and a constraint one. First dynamical equation has the form

$$
\begin{aligned}
& 2\left(\dot{H}_{b}+H_{b}^{2}\right)+2\left(\dot{H}_{c}+H_{c}^{2}\right)+2\left(\dot{H}_{d}+H_{d}^{2}\right)+2 H_{b} H_{c}+2 H_{b} H_{d}+2 H_{c} H_{d}+ \\
& +8 \alpha\left[\left(\dot{H}_{b}+H_{b}^{2}\right) H_{c} H_{d}+\left(\dot{H}_{c}+H_{c}^{2}\right) H_{b} H_{d}+\left(\dot{H}_{d}+H_{d}^{2}\right) H_{b} H_{c}\right]+\frac{16 \pi G}{c^{4}} w \varepsilon_{0}(a b c d)^{-(1+w)}=0
\end{aligned}
$$

where as usual $H_{i}=\frac{\dot{a}_{i}}{a_{i}}$, the rest of them could be obtained via cyclic index permutation. The constraint equation has a form

$$
2 H_{a} H_{b}+2 H_{a} H_{c}+2 H_{a} H_{d}+2 H_{b} H_{c}+2 H_{b} H_{d}+2 H_{c} H_{d}+24 \alpha H_{a} H_{b} H_{c} H_{d}=\frac{16 \pi G}{c^{4}} \varepsilon_{0}(a b c d)^{-(1+w)}
$$

\section{STATIONARY SOLUTIONS}

The system of equations (3) -(44) has solutions of a particular type in which all Hubble functions are constant (and, correspondingly, scale factors expand or contract exponentially), so, we call them as stationary for brevity. Anisotropic exponential solutions in Einstein-Gauss-Bonnet gravity with matter were particularly studied in [9] under some assumptions. Here we study maximally anisotropic case:

$$
g_{\mu \nu}=\left\{-1, e^{2 H_{1} t}, e^{2 H_{2} t}, \ldots, e^{2 H_{n} t}\right\}
$$

For solution to be a stationary one it is necessary that right-hand sides of field equations

$$
\alpha_{1} G_{\mu \nu}^{(1)}+\alpha_{2} G_{\mu \nu}^{(2)}=\frac{8 \pi G}{c^{4}} T_{\mu \nu}
$$


do not depend on time. This means that we need

$$
-(1+w) \sum_{i} H_{i}=0
$$

i. e. one of a two conditions should be satisfied:

1. $w=-1-$ matter is a cosmological constant; or

2. $\sum_{i} H_{i}=0-$ volume element is constant.

As in this section we have some results for an arbitrary number of spatial dimensions, we write down the equation of motion for a flat $(n+1)$-dimensional stationary case (i.e. neglecting all time derivatives):

$$
\begin{gathered}
\alpha_{1} \sum_{i<j} H_{i} H_{j}+12 \alpha_{2} \sum_{i<j<k<l} H_{i} H_{j} H_{k} H_{l}=\frac{8 \pi G}{c^{4}} \varepsilon_{0}, \\
\alpha_{1}\left(H_{j} \sum_{i} H_{i}-\sum_{i} H_{i}^{2}-\sum_{i<k} H_{i} H_{k}\right)+2 \alpha_{2}\left\{-2 \sum_{i \neq j} H_{i}^{2} \sum_{\substack{k, l \neq i \\
k<l}} H_{k} H_{l}+2 H_{j} \sum_{\substack{i, k \neq j \\
i \neq k}} H_{i}^{2} H_{k}+\right. \\
\left.+6 H_{j} \sum_{\substack{i, k, l \neq j \\
i<k<l}} H_{i} H_{k} H_{l}-6 \sum_{i<k<l<m} H_{i} H_{k} H_{l} H_{m}\right\}=\frac{8 \pi G}{c^{4}} w \varepsilon_{0} .
\end{gathered}
$$

For $w=-1$ we have investigated only $(4+1)$-dimensional space $(n=4)$ with $\alpha_{1}=1\left(\alpha \equiv \alpha_{2}\right)$ and have obtained the following solutions:

1.

$$
H_{1}=H_{2}=H, \quad H_{3}=H_{4}=-\frac{1}{4 \alpha H},
$$

where $H$ satisfies condition

$$
\frac{8 \pi G}{c^{4}} \varepsilon_{0}=\frac{1-4 \alpha H^{2}+16 \alpha^{2} H^{4}}{16 \alpha^{2} H^{2}} .
$$

2 .

$$
\begin{gathered}
H_{1} \equiv H \text { is arbitrary, } \quad H_{2}=H_{3}=\frac{\zeta}{\sqrt{-4 \alpha}}, \\
H_{4}=\frac{1-\zeta H \sqrt{-4 \alpha}}{\zeta \sqrt{-4 \alpha}+4 \alpha H}, \quad \frac{8 \pi G}{c^{4}} \varepsilon_{0}=-\frac{3}{4 \alpha},
\end{gathered}
$$

where $\zeta= \pm 1$. 
3.

$$
H_{1}=H, \quad H_{2}=\frac{z}{\alpha H}, \quad H_{3}=-\frac{1}{4 \alpha H}, \quad H_{4}=\frac{-16 \alpha^{2} H^{4}-1+16 \alpha H^{2} z-4 z}{4 \alpha H\left(-4 \alpha H^{2}+8 z+1\right)}
$$

where

$$
z=\frac{1-4 \alpha H^{2} \pm \sqrt{5\left(4 \alpha H^{2}-1\right)^{2}+16 \alpha H^{2}}}{8}
$$

and $H$ satisfies condition

$$
\frac{8 \pi G}{c^{4}} \varepsilon_{0}=\frac{16 \alpha^{2} H^{4}-4 \alpha H^{2}+1}{16 \alpha^{2} H^{2}}
$$

4.

$$
\begin{gathered}
H_{3}=-\frac{H_{1}+H_{2}}{2} \pm \sqrt{\frac{\left(H_{1}+H_{2}\right)^{2}\left(8 \alpha H_{1} H_{2}-3\right)+4 H_{1} H_{2}}{4\left(1+8 \alpha H_{1} H_{2}\right)}}, \\
H_{4}=-\frac{-4 \alpha H_{1} H_{2}\left(H_{1}^{2}+H_{2}^{2}\right)+H_{3}\left(H_{1}+H_{2}\right)\left(1+8 \alpha H_{1} H_{2}\right)+H_{1} H_{2}\left(1+4 \alpha H_{1} H_{2}\right)}{\left(1+8 \alpha H_{1} H_{2}\right)\left(H_{1}+H_{2}+H_{3}+12 \alpha H_{1} H_{2} H_{3}\right)},
\end{gathered}
$$

and $H_{1}, H_{2}$ satisfies condition

$$
\frac{8 \pi G}{c^{4}} \varepsilon_{0}=\frac{4\left(H_{1}^{2}+H_{2}^{2}+H_{1} H_{2}\right) \alpha H_{1} H_{2}}{1+8 \alpha H_{1} H_{2}} .
$$

5.

$$
\begin{gathered}
H_{1}=H_{2}=H, \quad H_{3}=z H \\
H_{4}=-\frac{H\left(288 \alpha^{2} H^{4} z+8 \alpha H^{2} z-2 z+52 \alpha H^{2}-1\right)}{96 \alpha^{2} H^{4} z-20 \alpha H^{2} z+8 \alpha H^{2}-z-2+1536 z H^{6} \alpha^{3}+288 \alpha^{2} H^{4}},
\end{gathered}
$$

where $z=-1 \pm \sqrt{\frac{8 \alpha H^{2}-2}{8 \alpha H^{2}+1}}$, and $H$ satisfies condition

$$
\frac{8 \pi G}{c^{4}} \varepsilon_{0}=\frac{12 H^{4} \alpha}{1+8 \alpha H^{2}}
$$

6.

$$
H_{1}=H_{2}=H_{3}= \pm \frac{1}{\sqrt{-4 \alpha}}, \quad H_{4} \text { is arbitrary, } \quad \frac{8 \pi G}{c^{4}} \varepsilon_{0}=-\frac{3}{4 \alpha}
$$


For $\sum_{i} H_{i}=0$ a general solution was investigated for arbitrary number of dimensions. If we express

$$
\begin{aligned}
24 & \sum_{i<j<k<l} H_{i} H_{j} H_{k} H_{l}=\sum_{i} H_{i} \sum_{j \neq i} H_{j} \sum_{k \neq i, j} H_{k} \sum_{l \neq i, j, k} H_{l}= \\
= & \sum_{i} H_{i} \sum_{j \neq i} H_{j} \sum_{k \neq i, j} H_{k}\left(-H_{i}-H_{j}-H_{k}\right)=-3 \sum_{i} H_{i}^{2} \sum_{j \neq i} H_{j} \sum_{k \neq i, j} H_{k}= \\
= & -3 \sum_{i} H_{i}^{2} \sum_{j \neq i} H_{j}\left(-H_{i}-H_{j}\right)=3 \sum_{i} H_{i}^{3} \sum_{j \neq i} H_{j}+3 \sum_{i} H_{i}^{2} \sum_{j \neq i} H_{j}^{2}= \\
= & -3 \sum_{i} H_{i}^{4}+3 \sum_{i} H_{i}^{2}\left(\sum_{j} H_{j}^{2}-H_{i}^{2}\right)=-6 \sum_{i} H_{i}^{4}+3\left(\sum_{i} H_{i}^{2}\right)^{2}
\end{aligned}
$$

and similarly for other products of Hubble parameters, we get the equations in the form

$$
\begin{aligned}
& -\alpha_{1} \sigma_{2}+3 \alpha_{2}\left(\sigma_{2}^{2}-2 \sigma_{4}\right)=2 \varkappa_{0}, \\
& -\alpha_{1} \sigma_{2}+2 \alpha_{2}\left(\sigma_{2}^{2}-2 \sigma_{4}\right)=(1+w) \varkappa_{0},
\end{aligned}
$$

where

$$
\sigma_{2} \equiv \sum_{i} H_{i}^{2}, \quad \sigma_{4} \equiv \sum_{i} H_{i}^{4}, \quad \varkappa_{0} \equiv \frac{8 \pi G}{c^{4}} \varepsilon_{0} .
$$

If $\alpha_{1} \neq 0$ and $\alpha_{2} \neq 0$ then we obtain that the general solution is any set of $H_{i}, i=1, \ldots n$, that satisfies the following conditions:

$$
\begin{aligned}
& \sum_{i} H_{i}=0, \\
& \sum_{i} H_{i}^{2}=\frac{1-3 w}{\alpha_{1}} \varkappa_{0}, \\
& \sum_{i} H_{i}^{4}=\frac{w-1}{2 \alpha_{2}} \varkappa_{0}+\frac{(1-3 w)^{2}}{2 \alpha_{1}^{2}} \varkappa_{0}^{2} .
\end{aligned}
$$

From this we can obtain a particular case of [9] where $H_{1}=H_{2}=H_{3}, H_{4}=H_{5}=\ldots=H_{n}$.

It is interesting to note that from equations (8) in the case $\alpha_{1}=0$ and $\varepsilon_{0}=0$ we have only one condition to be satisfied

$$
\sum_{i} H_{i}^{4}=\frac{1}{2}\left(\sum_{i} H_{i}^{2}\right)^{2}
$$

and that is in agreement with [10]. It can be also easily seen than the only case for a matter other than cosmological constant allowing a stationary solution in the pure Gauss-Bonnet gravity is of $w=1 / 3$ type. In this case the condition is 


$$
\left(\sum_{i} H_{i}^{2}\right)^{2}-2 \sum_{i} H_{i}^{4}=\frac{2 \varkappa_{0}}{3 \alpha_{2}}
$$

One can easily see that (10) turns into (9) if $\varepsilon_{0}=0$. These two (9, 10) are the only special solutions of (맘 if $\alpha_{1}=0$.

\section{NUMERICAL STUDIES}

Before describing regimes let us make some notes regarding our strategy. First of all, just like in previous papers where we numerically studied (4+1)- and (5+1)-dimensional Bianchi-I models [11, 12], we consider only models with positive initial $\sum H_{i}$ (and in this section we again use dynamical $H_{i}=H_{i}(t)$ in contrast with the previous section where due to parametrization used our Hubble function were constant). In $(4+1)$ dimensions we specify 3 initial Hubble functions and calculate 4th using the constraint equation (44). This expression has a form

$$
H_{d}^{(0)}=-\frac{2 H_{a}^{(0)} H_{b}^{(0)}+2 H_{a}^{(0)} H_{c}^{(0)}+2 H_{b}^{(0)} H_{c}^{(0)}-\rho_{0}}{2 H_{a}^{(0)}+2 H_{b}^{(0)}+2 H_{c}^{(0)}+24 \alpha H_{a}^{(0)} H_{b}^{(0)} H_{c}^{(0)}},
$$

and here (and later) we use definition for $\rho_{0}$ (and later for $\rho$ ) to simplify notations: $\rho_{0}=16 \pi G \varepsilon_{0} / c^{4}$. Our goal is to study numerically the influence of non-zero matter density on the evolution scenario. We fix 3 Hubble parameters and change $\rho$ and the equation of state parameter $w$. In this approach two different possibilities exist depending on the sign of denominator. If it is positive, we have no restriction on the initial value of matter density $\rho$. If the denominator is negative, some maximal value of initial density exists (that maximal value satisfy $\sum H_{i}^{(0)}=0$ ).

Solving $\dot{H}_{i}=0$ system with respect to $(w, \rho)$ gives us values $\left(w_{s t}, \rho_{s t}\right)$ for stationary regime:

$$
w_{s t}=\frac{H_{a}^{2}+4 \alpha H_{b} H_{a} H_{c}^{2}+4 \alpha H_{c} H_{b} H_{a}^{2}+4 \alpha H_{c} H_{a} H_{b}^{2}+H_{c}^{2}+H_{b} H_{a}+H_{c} H_{a}+H_{b}^{2}+H_{c} H_{b}}{H_{b}^{2}+H_{c}^{2}+H_{a}^{2}+H_{c} H_{b}+H_{c} H_{a}+H_{b} H_{a}+12 \alpha H_{c} H_{b} H_{a}^{2}+12 \alpha H_{c} H_{a} H_{b}^{2}+12 \alpha H_{b} H_{a} H_{c}^{2}},
$$

$$
\rho_{s t}=-2 H_{b}^{2}-2 H_{c}^{2}-2 H_{a}^{2}-2 H_{c} H_{b}-2 H_{c} H_{a}-2 H_{b} H_{a}-24 \alpha H_{c} H_{b} H_{a}^{2}-24 \alpha H_{c} H_{a} H_{b}^{2}-24 \alpha H_{b} H_{a} H_{c}^{2},
$$

and that regime corresponds to the one, described analytically in the previous section. 
Table. Possible trajectories for vacuum (4+1)-dimensional GR+GB model

\begin{tabular}{c|c|c|c}
\hline From & To & Design. & Avail. \\
\hline Standard singularity & Kasner & I & + \\
& Recollapse & II & + \\
& Non-standard sing. & III & - \\
\hline Non-standard. sing. & Kasner & IV & + \\
& Recollapse & V & - \\
& Non-standard sing. & VI & + \\
\hline
\end{tabular}

\section{A. Singular vacuum regimes}

We start with listing of possible regimes in (4+1)-dimensional vacuum Bianchi-I model. There are two possible outcomes for the past evolution - standard and non-standard singularities, and three for the future evolution - non-standard singularity, Kasner regime and recollapse. One can naively suppose that all six possible combinations of "past" and "future" regimes are occuring, but the reality is much more complicated. We summarized the trajectories in Table.

As it was noted in [14], type-I regime require fine-tuning of initial Hubble parameters, namely, three of them should be equal to each other. Recently [15] it was noted that sometimes the equality of two Hubble parameters is enough to achieve this type of regime.

In contradiction with [14], we failed to find type-III transitions. Authors of paper [14] claim they found them (around 1.5\% of total number of trajectories), so keeping in mind possible numerical errors as well as different criteria to stop the integration process we can conclude that this regime is very rare if exists at all (see also [15] for possible reasons behind this).

\section{B. Regimes in presence of matter}

Since distinguishing of initial conditions with different signs of denominator in Eq. (11) is important for us, we treat these two cases separately. A typical transition diagram on the $(\rho, w)$ plane for the case of positive denominator is given in Fig. 1 (to be precise, it corresponds to the vacuum type-II case with positive denominator). White region corresponds to the type-II behavior, different grey regions correspond to VI, IV, III and I with increase of darkness of grey; they also denoted in figure.

From this figure we can see that type III evolution (which we have not seen in the vacuum case) 


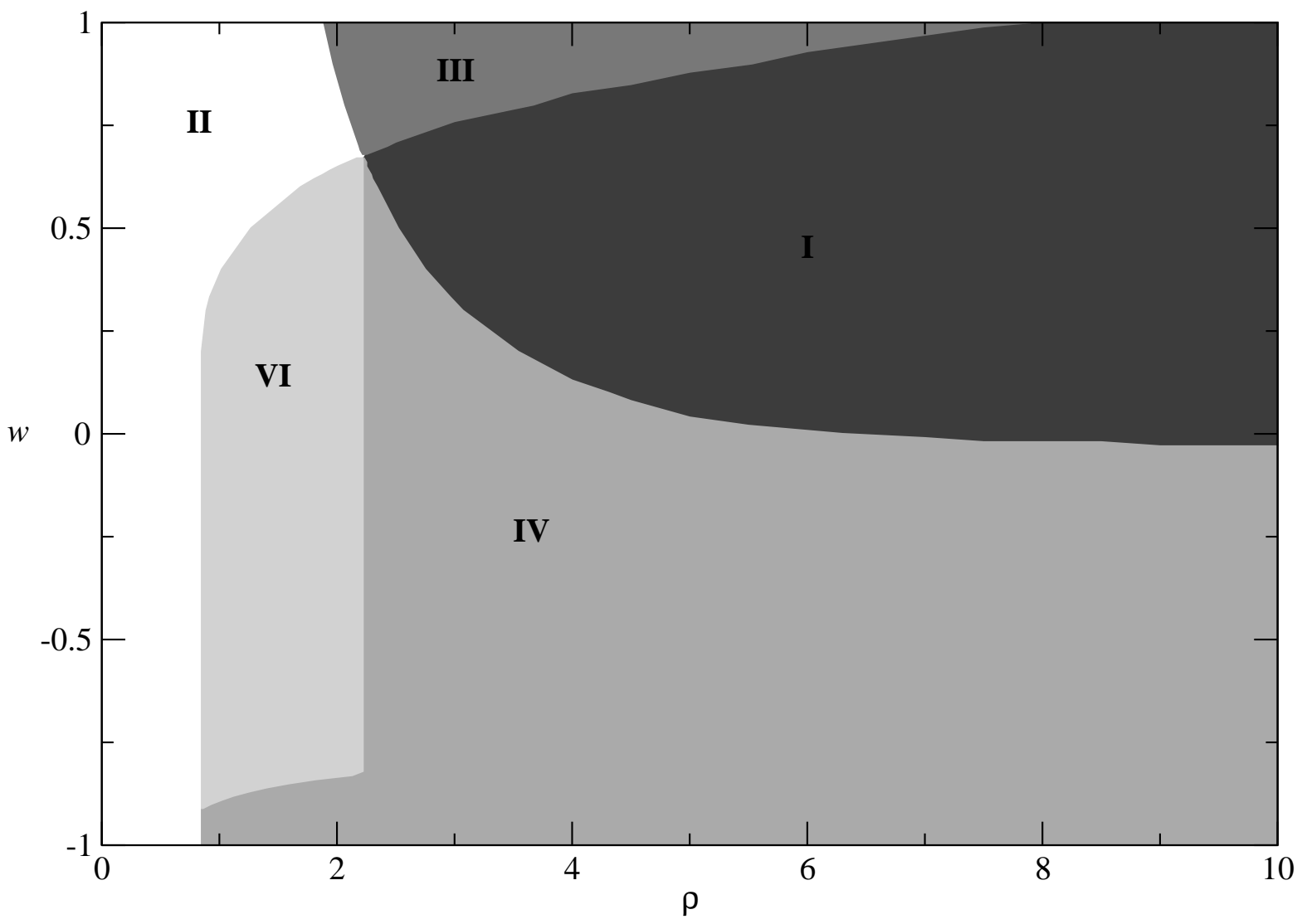

FIG. 1: An example trajectories map in $(\rho, w)$ coordinates for II case with positive denominator. White region corresponds to the type-II transition, different grey regions correspond to VI, IV, III and I with increase of darkness of grey; they also denoted in figure.

and type I evolution (which occurs in the vacuum case only under very specific initial conditions) both appear as possible scenarios without any severe fine-tuning of initial conditions. As a result, all 6 possible scenarios listed in the Table 1, are possible when matter is taken into account.

In the transition diagram for the case of negative denominator we have the stationary regime at the right limit of the diagram if corresponding $w_{s t}$ lies in the physical zone $w_{s t}>-1$. In Fig. 2 as a way of example we present a typical example for negative $w_{s t}$ (that is type-VI case with negative denominator). The situation at the limit line $\rho=\rho_{s t}$ is as follows.

If $w>w_{s t}$ and $w \rightarrow w_{s t}+0$ the "lifetime" of the type-II regime is increasing and asymptotically reaches infinity when $w=w_{s t}$. In Fig. 3 we presented the transition in question: Fig. 3(a) represents Hubble functions versus time for standard type-II regime (standard singularity to recollapse); one can see in Fig. 3(b), where we plot $\left(H_{i}(t)-H_{a}(t)\right) / H_{a}(t)$ that the second singularity (that 


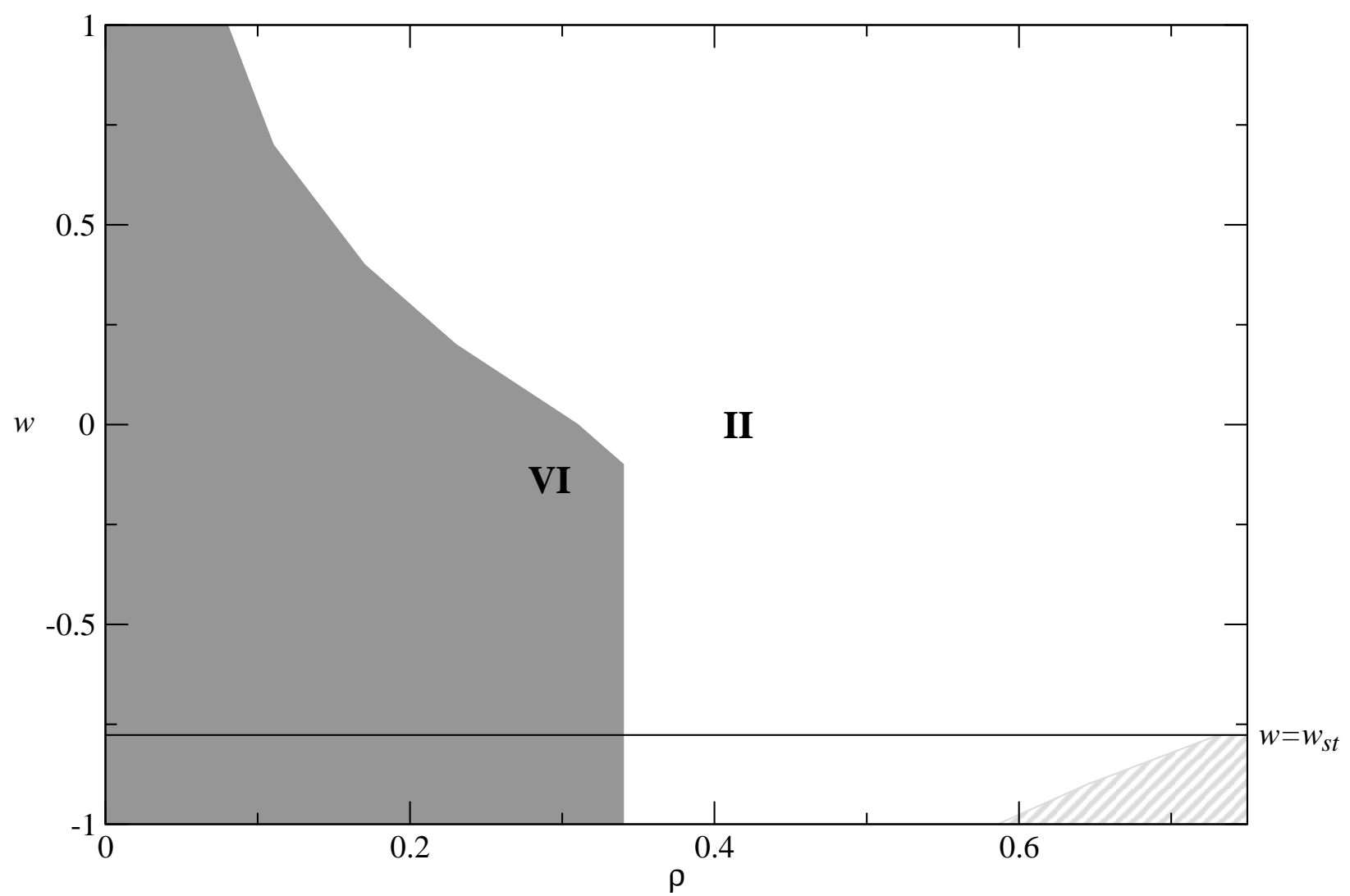

FIG. 2: An example trajectories map in $(\rho, w)$ coordinates for VI case with negative denominator. Stationary regime occur at $w=w_{s t}$ and $\rho=\rho_{s t}$, i.e. at the intersection of $w=w_{s t}$ with right $\rho$-boundary. Stroked region in the right-bottom corner denotes periodical trajectories.

corresponds to recollapse) is isotropic one. With $w \rightarrow w_{s t}+0$ (and $\rho \rightarrow \rho_{s t}$ ) the situation changes according to Fig. 3(c); second singularity is still isotropic (Fig. 3(d)). Finally, when $\rho=\rho_{s t}$ and $w=w_{s t}$, the stationary regime is reached (Fig. 3(e)).

If $w<w_{s t}$ we have quite different behavior. Namely, it becomes periodical; in Fig. 4 we presented it. All through Fig. 4 we used the same value for density $\rho \approx \rho_{s t}$ and decreased equation of state $w$ from $w \approx w_{s t}-0$ in Fig. 4(a) down to $w=-1$ in Fig. 4(e). One can see that with decrease of the equation of state the period and amplitude of oscillations are first increasing first, and then smoothly turn into quasi-stationary regime at $w=-1$. This means that small homogeneous perturbation of stationary regime does not necessary destroys nonsingular behavior, but turn it to periodic oscillations near the stationary point. If $\rho$ is less enough than $\rho_{s t}$ then the described above periodical regime is not triggered and we have singular regime for any $w$. 

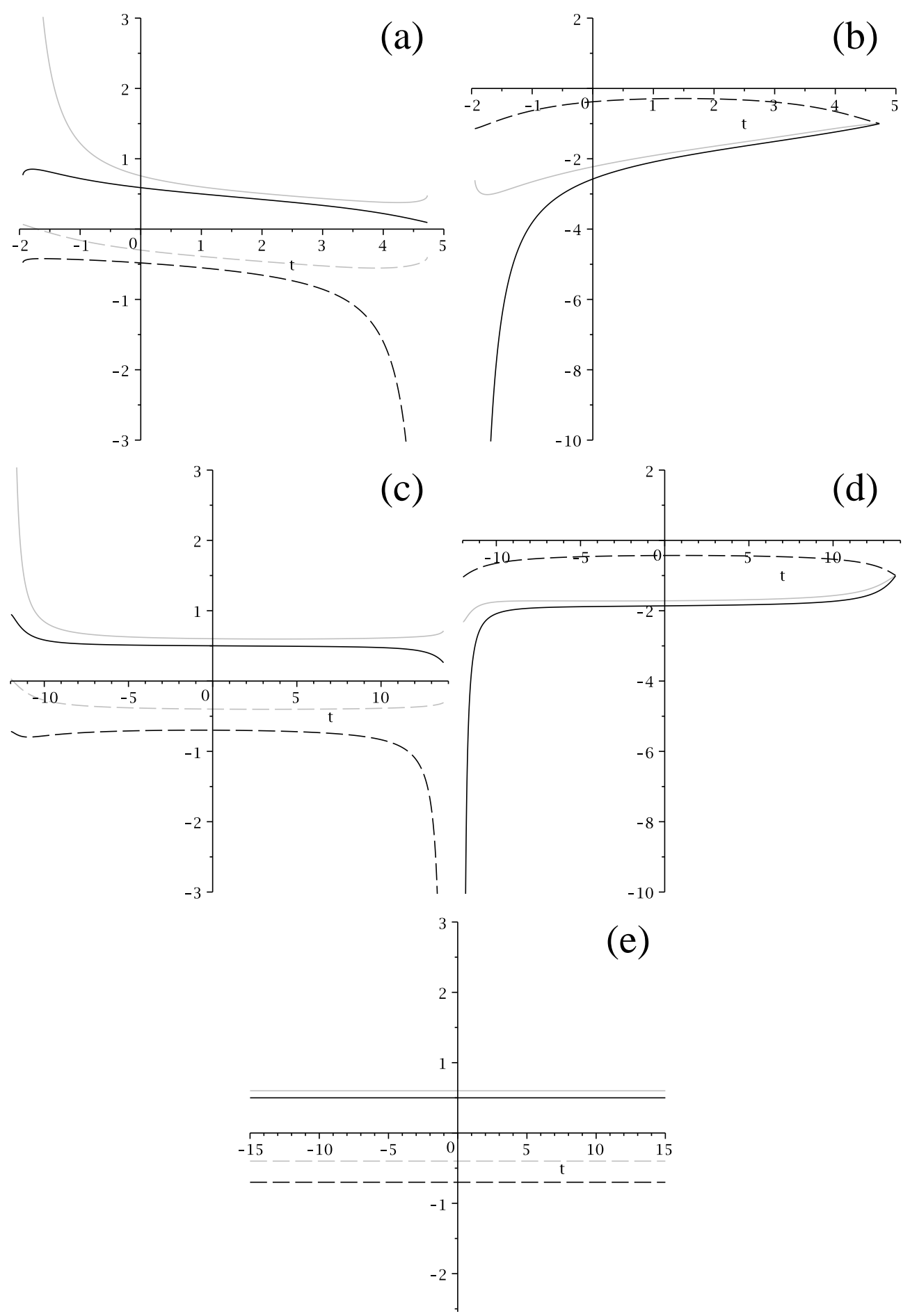

FIG. 3: The transition from standard type-II to stationary regime with $w_{s t}<0$ and $w \rightarrow w_{s t}+0$. Hubble functions in the standard type-II regime are presented in (a); relative differences $\left(H_{i}(t)-H_{a}(t)\right) / H_{a}(t)$ in (b) demonstrate that the second singularity (that corresponds to recollapse) is isotropic one. With $w \rightarrow w_{s t}+0$ the situation changes according to (c): the "lifetime" is increasing with "central" part looks pretty flat; the second singularity remains isotropic one (d). Finally, when $\rho=\rho_{s t}$ and $w=w_{s t}$, the regime becomes stationary one (e). 

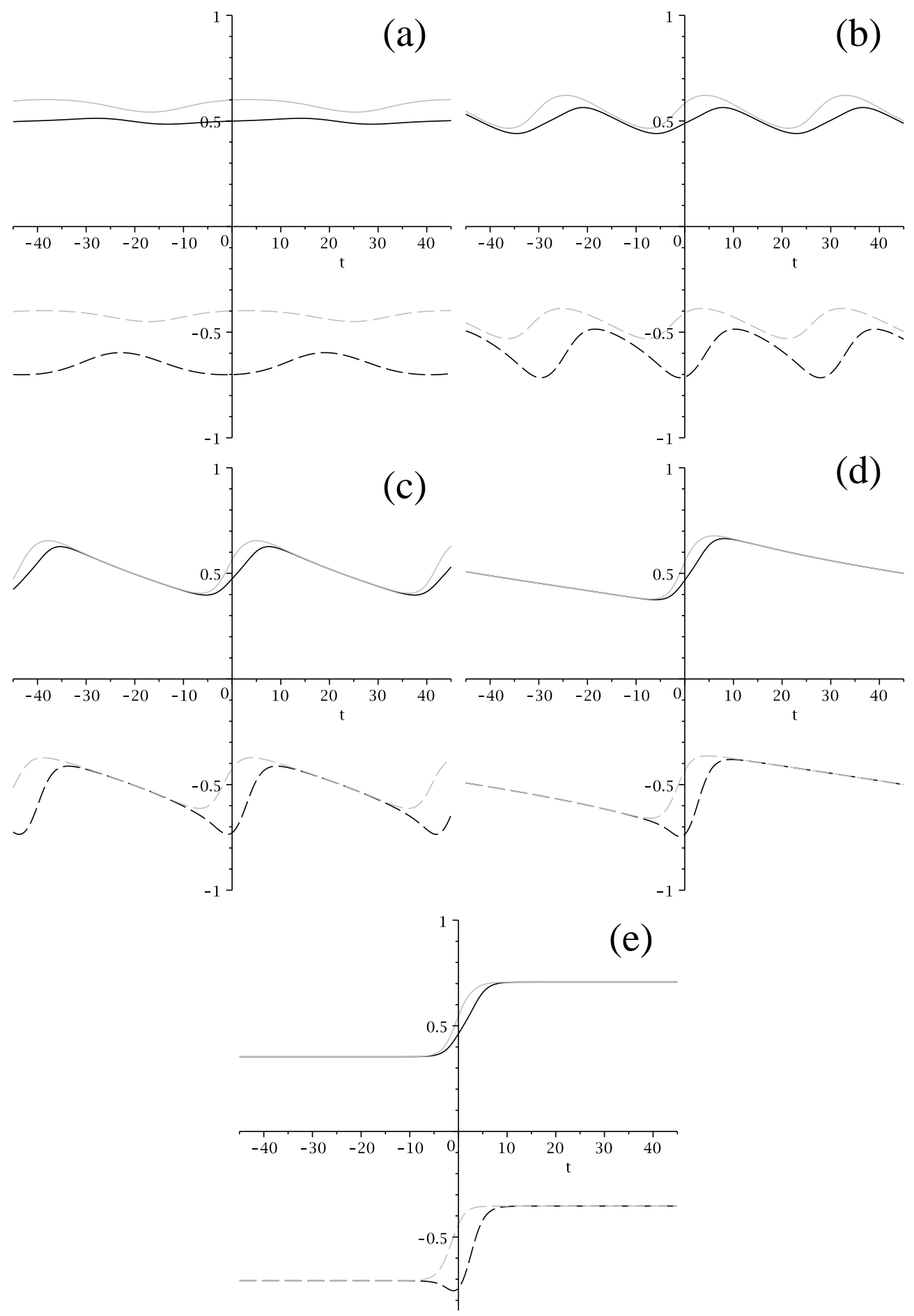

FIG. 4: The transition from standard type-II to stationary regime with $w_{s t}<0$ and $w \rightarrow w_{s t}-0$. We used the same $\rho \approx \rho_{s t}$ for all panels (a)-(e) and decreased the equation of state from $w \approx w_{s t}-0$ in panel (a) down to $w=-1$ in panel (e). See text for details. 


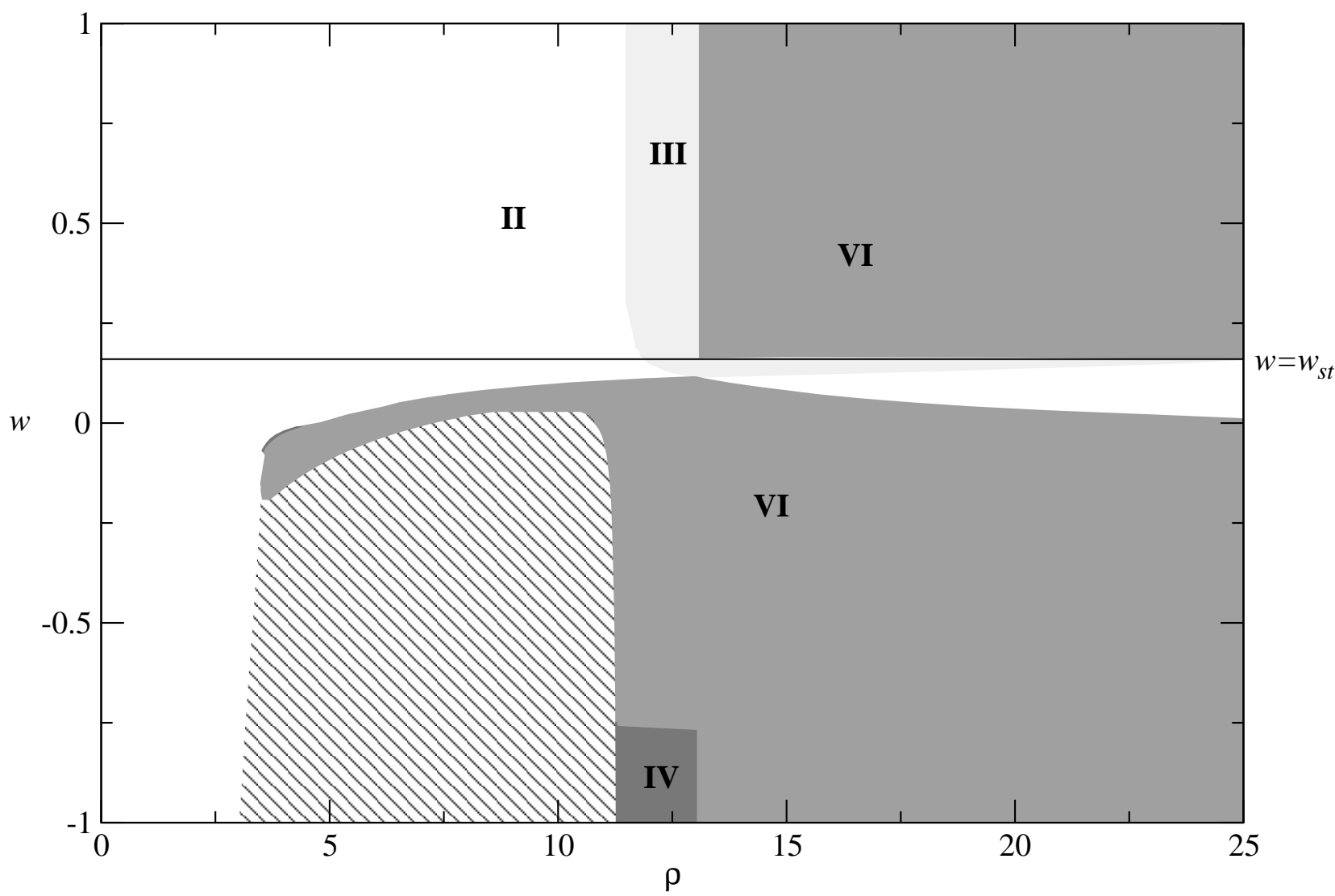

FIG. 5: An example map of transitions in $(\rho, w)$ coordinates for $1 / 3>w_{s t}>0$ case. White corresponds to type-II transitions; stroked white - periodical trajectories as described in $w_{s t}<0$ subsection; light grey type-III trajectories; grey - type-VI and dark grey - type-IV.

In case of $w_{s t}>0$ the situation is different from described above. There are no periodic regimes in the vicinity of the stationary point. Any small perturbations of initial conditions needed for this solution ultimately leads to singular behavior. An example for regime transitions diagram for this case is plotted in Fig. 5. In general, we have not seen any periodic oscillations numerically with $w>0$.

\section{CONCLUSIONS}

We have considered dynamics of a flat $(4+1)$-dimensional anisotropic Universe filled with an ordinary matter in Gauss-Bonnet gravity. Regarding singular regimes, we have founded that any of possible 6 different regimes (with 2 possible initial points in standard or nonstandard singularities, and 3 possible future outcomes - recollapse, nonstandard singularity or low-curvature Kasner 
solution) can be realized without severe fine-tuning of initial conditions. This means that some constraints of $(4+1)$-dimensional dynamics in Gauss-Bonnet gravity found for vacuum regimes are lifter when matter is taken into account. Full description of all transition in both vacuum and matter cases is given in [15].

We have found also two nonsingular regimes - a stationary one with constant values of Hubble parameters and zero volume expansion rate (so, $\left.\sum H_{i}=0\right)$ which have been found analytically for an arbitrary number of dimensions and oscillatory regime founded numerically in the vicinity of the stationary regime for $(4+1)$-dimensional case. In our numerical studies we have seen this latter regime only for $w<0$.

\section{Acknowledgements}

This work was partially supported by RFBR grant 08-02-00923.

[1] F. Müller-Hoissen, Phys. Lett. 163B, 106 (1985).

[2] J. Madore, Phys. Lett. 111A, 283 (1985); J. Madore, Class. Quant. Grav. 3, 361 (1986); F. MüllerHoissen, Class. Quant. Grav. 3, 665 (1986).

[3] N. Deruelle, Nucl. Phys. B327, 253 (1989).

[4] T. Verwimp, Class. Quant. Grav. 6, 1655 (1989); G. A. Mena Marugán, Phys. Rev. D 42, 2607 (1990); ibid. 46, 4340 (1992).

[5] N. Deruelle and L. Fariña-Busto, Phys. Rev. D 41, 3696 (1990).

[6] D. Lovelock, J. Math. Phys. 12, 498 (1971).

[7] R. Metsaev and A. Tseytlin, Nucl.Phys. B293, 385 (1987).

[8] K. Bamba, S. Odintsov, L. Sebastiani, and S. Zerbini, arXiv:0911.4390,

[9] I. V. Kirnos and A. N. Makarenko, arXiv:0903.0083.

[10] V. D. Ivashchuk, arXiv:0910.3426,

[11] S.A. Pavluchenko and A.V. Toporensky, Mod. Phys. Lett. A24, 513 (2009) // arXiv:0811.0558

[12] I.V. Kirnos, A.N. Makarenko, S.A. Pavluchenko, and A.V. Toporensky, arXiv:0906.0140

[13] S.A. Pavluchenko, Phys. Rev. D80, 107501 (2009) // arXiv:0906.0141

[14] R. Chingangbam, M. Sami, P. V. Tretyakov, and A.V. Toporensky, Phys. Lett. B661, 162 (2008) // arXiv:0711.2122

[15] S.A. Pavluchenko, arXiv:1003.4892. 